\title{
First experiences with Personal Networks as an enabling platform for service providers
}

\author{
F.T.H. den Hartog, M.A. Blom, C.R. Lageweg, \\ M.E. Peeters, J.R. Schmidt, R. van der Veer, \\ A. de Vries, M.R. van der Werff \\ TNO \\ Brassersplein 2, P.O. Box 5050 \\ 2600 GB Delft, The Netherlands \\ Frank.denHartog@tno.nl
}

\author{
Q. Tao, R.N.J. Veldhuis \\ University of Twente, EEMSC, Signals \& Systems \\ P.O. box 217, 7500 AE, Enschede, The Netherlands \\ N.H.G. Baken, F. Selgert \\ KPN \\ Maanplein 55, P.O. Box 30000 \\ 2600 GA Den Haag, The Netherlands
}

\begin{abstract}
By developing demonstrators and performing smallscale user trials, we found various opportunities and pitfalls for deploying Personal Networks (PNs) on a commercial basis. The demonstrators were created using as many as possible legacy devices and proven technologies. They deal with applications in the health sector, home services, tourism, and the transportation sector. This paper describes the various architectures and our experiences with the end users and the technology. We conclude that context awareness, service discovery, and content management are very important in PNs and that a Personal Network Provider role is necessary to realize these functions under the assumptions we made. The PNPay Travel demonstrator suggests that $P N$ service platforms provide an opportunity to develop true trans-sector services.
\end{abstract}

\section{INTRODUCTION}

Ever more electronic devices appear in people's various private domains (home, car, office, workplace, the personal operating space, etc.) that contain some networking functionality and form local networks ("private networks") with a geographically limited size. Examples are home networks, Personal Area Networks (PANs) and company networks, but also more innovative networks such as Streetlight [1]. These networks enable the user's devices to share content, data, applications, and resources with each other, and communicate with the Internet, either directly or via a gateway, for instance the Residential Gateway (RG) [2]. However, the technology for sharing between the private networks is still very limited. This is caused by the heterogeneity in technology used in the various networks, the mobility of some of these networks (e.g. the PAN and the incar network), and the lack of a unified access procedure ('single login').

A Personal Network (PN) is envisaged as the next step in achieving unlimited communication between people's electronic devices [3]. It comprises the technology needed to

The work presented in this article has been carried out in the collaborative Freeband Communication technology program which is supported by the Dutch Ministry of Economic Affairs. interconnect the various private networks of a single user seamlessly, at any time and at any place, even if the user is highly mobile. A PN guarantees the user seamless (remote) access to any personal device, service and content. He perceives access to all his content and services as if they were physically present in his vicinity. A schematic view of a PN is given in Fig. 1. It is drawn as an overlay that hides the underlying technology and business complexity from the user.

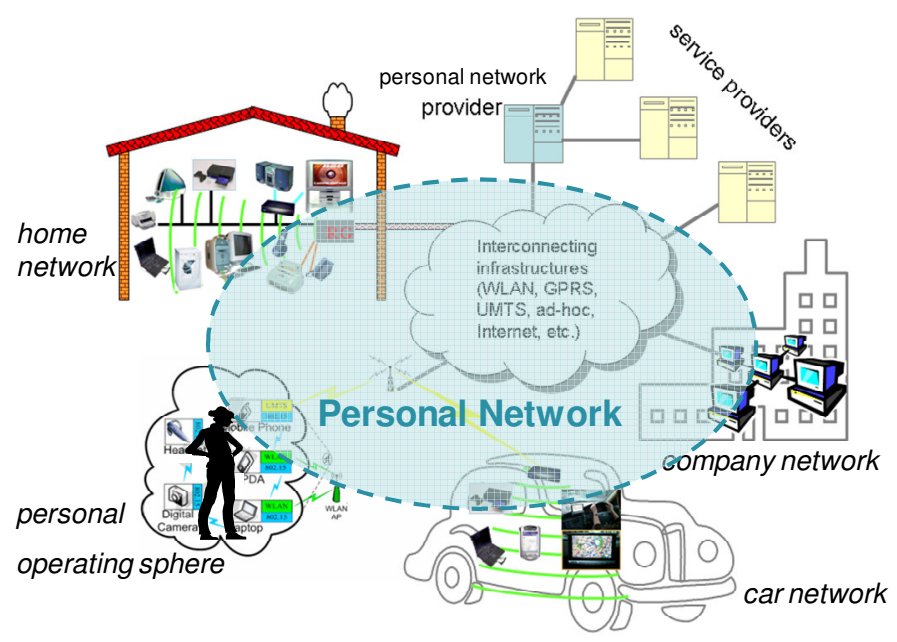

Figure 1. A Personal Network (PN) is a personalized overlay over multiple network domains. The Personal Network Provider enables the PN, and offers (third party) services to the PN user.

In the Freeband PNP2008 project [4] we investigate how PNs can be realized using as many as possible legacy devices and proven technology. The reason why we chose this approach lies in the assumption that commercial realization of PNs will happen sooner if only a minimum of requirements is put on the end-user devices participating in a PN. This choice leads to severe consequences for the PN architecture:

- The architecture has a centralized character, with an omnipotent central server that communicates with relatively intelligent private network gateways and 
presents an abstraction of the PN to third-party service providers. The gateways control the legacy devices in the private networks involved in the PN.

- Next to Internet Protocol version 6 (IPv6), also IPv4 should be considered. Especially in home networks, IPv4 will dominate for quite some time into the future.

- Next to IP-networks, also non-IP networks should be considered, such as Bluetooth [5] PANs and home automation networks such as LonWorks [6] and Konnex [7].

- Migration scenarios to more advanced PN architectures should be studied and taken into account.

- It should be accepted that on the short term the PN functionality offered to the user will have some limitations.

An important factor for enabling such a $\mathrm{PN}$ is the provisioning and management of the central PN server(s) as mentioned in the first bullet. We assign this task to a so-called Personal Network Provider (PNP) [8]. The PNP is a new business role that can be fulfilled by a commercial party such as a telecom operator or an Internet service provider. Alternatively, an expert user who knows how to run and maintain servers in one of his private network domains can be his own PNP. The PNP is the service provider enabling the $\mathrm{PN}$, offering third-party services, and providing an operational environment to manage user-, service-, content- and networkrelated issues of the PN. For third-party service providers, the PNP might act as a one-stop shop for providing their services to the PN user. Basically, the PNP solves the short-comings of current technology for non-expert users, ensuring ease of use for PN functionality.

To show how PNs can improve current services and enable new services for the end user, PNP2008 has taken a practical approach. Every year, we develop a demonstrator and perform small-scale user trials. We use the results to expand our vision on PN architecture and business opportunities and then produce a more advanced demonstrator the year after. This paper focuses on our experiences with four PNP2008 demonstrators and two user trials. The first demonstrator is called Medicam and shows the use of PNs in a health care environment. The Always-at-Home demonstrator is designed around remote access to home services. The core of the third demonstrator, MarcoPolog, is a content management system applied to tourist services. The latest demonstrator is PNPay Travel and shows how PNs can overcome the barrier between the public transport sector and the private transport sector (cars, trucks, etc.) by integrating billing services and by combining road- and railtransport route planning. In choosing different sectors, we also investigated how generic PNs can be deployed as a serviceenabling platform. User trials have been performed with the Medicam and MarcoPolog demonstrators and the results of the trials will be discussed briefly.

\section{MEDICAM}

Our experiences with the Medicam demonstrator have been documented in [9] and are summarized below. Medicam shows how PNs can improve the multimedia communication between the operating room in hospitals and remote peers and students. It focuses on how to search and connect to contextrelevant information within a increasingly complex professional environment.

In medical emergency operations, the medical staff involved has limited time to make a diagnosis and to collect all the information needed. Quite often, therefore, the operating surgeon has to consult a colleague by physically leaving and reentering the operating theatre, which is fairly time consuming because of the strict hygiene regulations. A PN might improve the communication significantly. In the Medicam demonstrator, the PN consists of the PANs of the surgeon and his remote peers (containing various mobile communication devices), the local area network of the emergency room (containing controllable high quality cameras), and the local network of the peers (containing any type of screen). This setup is drawn in Fig. 2.

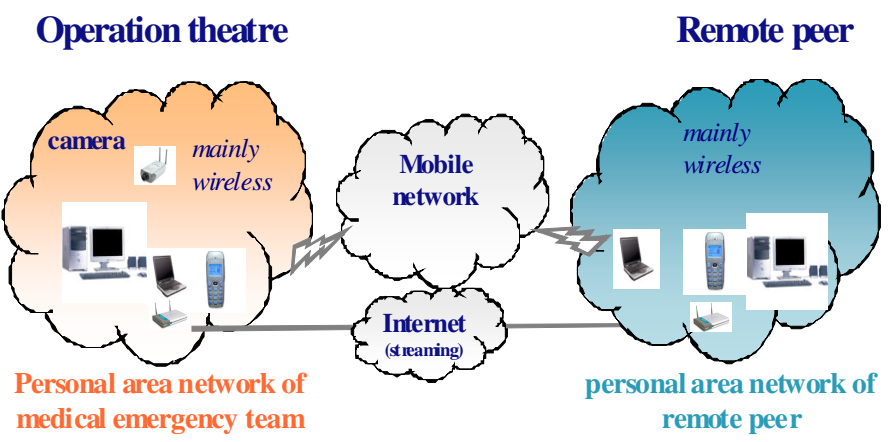

Figure 2. Schematic overview of the Medicam demonstrator set-up.

We assume a medical emergency team working in the operation theatre. A number of controllable digital video cameras are recording the scene. The cameras and the streaming server become part of the surgeon's PAN as soon he enters the operating theatre. On demand, the surgeon can choose to deliver the live streaming content to the outside world. For consulting peers around the world, an invitation phone call is made to a group of relevant peers based on their expertise and presence. The invited peers receive a message with the invitation to join the surgeon's PN. From that moment on the medical emergency team and the remote group members are able to talk to each other about the operation. The operating team then sends a message to the peers containing a web address and login details, allowing the group members to access the live streaming video on their mobile phone or on any local device with a screen being (temporarily) absorbed into their PAN, such as the home TV. Moreover, the surgeon can delegate the control (pan, tilt, zoom) of one or more cameras to one of the invited peers to have an optimal interaction.

The architecture of the demonstrator can be described as follows. The emergency room has a wireless local area network, based on the IEEE 802.11a standard, which is connected to the Internet by means of a router/gateway. The wireless network consists of a high quality networked camera with a streaming server and a control interface. It also contains a PC that can show the camera image and manages the camera 
so that it can be automatically discovered by other devices that enter the theatre. This PC can also be used to collect other health data of the patient. The PAN of the surgeon consists of a General Packet Radio Services (GPRS) and Bluetooth enabled mobile phone and a Bluetooth and IEEE 802.11a enabled laptop or Personal Digital Assistant (PDA). The laptop/PDA is used to discover the camera (by using the Zeroconfig auto-discovery and -configuration protocol standardized by the Internet Engineering Task Force (IETF) [10]). The phone is used for inviting group members and setting up a group conversation by using a Push-to-Talk service [11]. For Push-to-Talk an extra server is needed that is deployed and maintained by e.g. the PNP. Via the Bluetooth connection between the phone and the laptop, the camera can be controlled and the camera details (web address, login details) can be retrieved and sent to the message server. This message server is based on the Simple Object Access Protocol and Remote Procedure Call (SOAP-RPC [12]), and is also to be deployed and maintained by the PNP.

Each peer has a PAN containing a similar mobile phone and laptop/PDA. The mobile phone is used for the Push-toTalk session, and the IEEE 802.11a enabled laptop/PDA is used for discovering a screen with a video player nearby and for receiving the camera instructions from the mobile phone. The screen might be connected to the Internet by its own broadband connection (the home network or the hospital network). In that case the camera images can be routed to the screen via the broadband fixed network. If the screen is standalone, the images might be streamed via the much slower GPRS and Bluetooth connection. In that case, some content adaptation should be performed by, for instance, the PNP.

On the whole, Medicam proves to be working quite reliably. The most important limitation is encountered when there are many (e.g. tens) Bluetooth-enabled devices near the mobile phone of the surgeon or the peer. It then takes a very long time (up to minutes) for the surgeon's phone to find and connect to the correct devices. For the peer, it may take even longer to discover an alternate screen for displaying the streaming video. Also, Bluetooth does not provide for a shortcut algorithm to reconnect whenever the connection is temporarily lost.

We have investigated the need and user experience of PNs in hospitals by performing user trials with tens of doctors and nurses working in hospital departments with (potentially) high ICT usage and high relevance of teamwork and multi-location peer-to-peer counseling. The test method we used is a combination of individual context interviews, scenario and requirements verification, guided experimenting with the demonstrator and concluding focus groups (workshops). We found that Personal Networks in general and Medicam in particular, are conceived as especially useful in the following cases:

- A PN enables personalized access to electronic patient files at any time and place, improving the quality of patient transfers by improving information exchange between hospitals, departments, and other health care institutes.

- PNs can support the education of student surgeons. At the moment, it is difficult to show specific practices and operations in a hospital to a large number of students interactively at the same time.

- PNs enable remote supervision of several operations simultaneously by a senior surgeon, even including the activities in an ambulance.

- Regional specialist meetings or department meetings can be attended remotely, while cases can be discussed using diverse multimedia applications and networked devices that are present at the different locations.

\section{ALWAYS AT HOME}

The Always at Home demonstrator is developed to show the added value of PNs for end users in their daily life. It is based on a user scenario describing an office worker traveling home. While traveling, the user listens to his mp3 player using his headset. When he is called on his mobile phone, he does not touch the phone but uses the headset from his mp3 player to receive the call. The $\mathrm{mp} 3$ player automatically pauses during the phone call. The user consults his PDA to check out the content on his mp3 player, as well as the music stored on his $\mathrm{PC}$ at home. He can also use his PDA to program is Personal Video Recorder (PVR) and to check who is at home at the moment. When somebody rings the doorbell, the doorbell system checks whether somebody is at home. If that is not the case, it streams video data from the front door camera to the PDA in the user's PAN, and the user can choose to open the door remotely. When the user arrives at home, he switches on the television and watches a movie. Again somebody rings the doorbell. The doorbell system now streams the video data provided by the front door camera to the TV in the home network, after the PVR has paused the movie. An extended version of this scenario is filmed and can be viewed in [4]. The $\mathrm{PN}$ architecture supporting the extended scenario is described in [13].

The architecture of the Always at Home demonstrator is shown in Fig. 3. The PAN consists of a commercially available Bluetooth-enabled mp3 player, a headset, a Bluetooth-enabled mobile phone and a IEEE 802.11b enabled PDA. Unfortunately, the available devices do not support enough Bluetooth profiles to make the PAN completely wireless. The mp3 player does not support the Advanced Audio Distribution Profile (A2DP) profile, so the headset is hard wired to the player. It does not support the file transfer profile either. Therefore, the files discovered by the PDA can only be transferred to the player using a Universal Serial Bus (USB) cable.

The home network contains an RG, a doorbell with camera, a Radio Frequency IDentity (RFID [14]) reader, a PVR and a TV. The doorbell is connected to the RG by means of a serial (RS232) cable and controlled with the Batibus protocol [7]. The RFID reader is also connected to the RG by means of RS232. The PVR is connected to the RG via IEEE $802.11 \mathrm{~b}$ and to the TV with analogue cabling. The home network and the PAN are connected with each other via a single IEEE 802.11b sub-network.

To use the PDA for discovering mp3 files in the home network and transfer them to the mp3 player, a network 
mapping and a desktop item to the hard disk of the RG are made on the PDA. The RG hard disk contains the mp3 files. When the PDA is connected to the mp3 player, the flash memory of the mp3 player is shown in the PDA's file explorer as 'removable device'. Mp3 files can then be copied from the $\mathrm{RG}$ hard disk to the mp3 player's flash memory via the PDA.
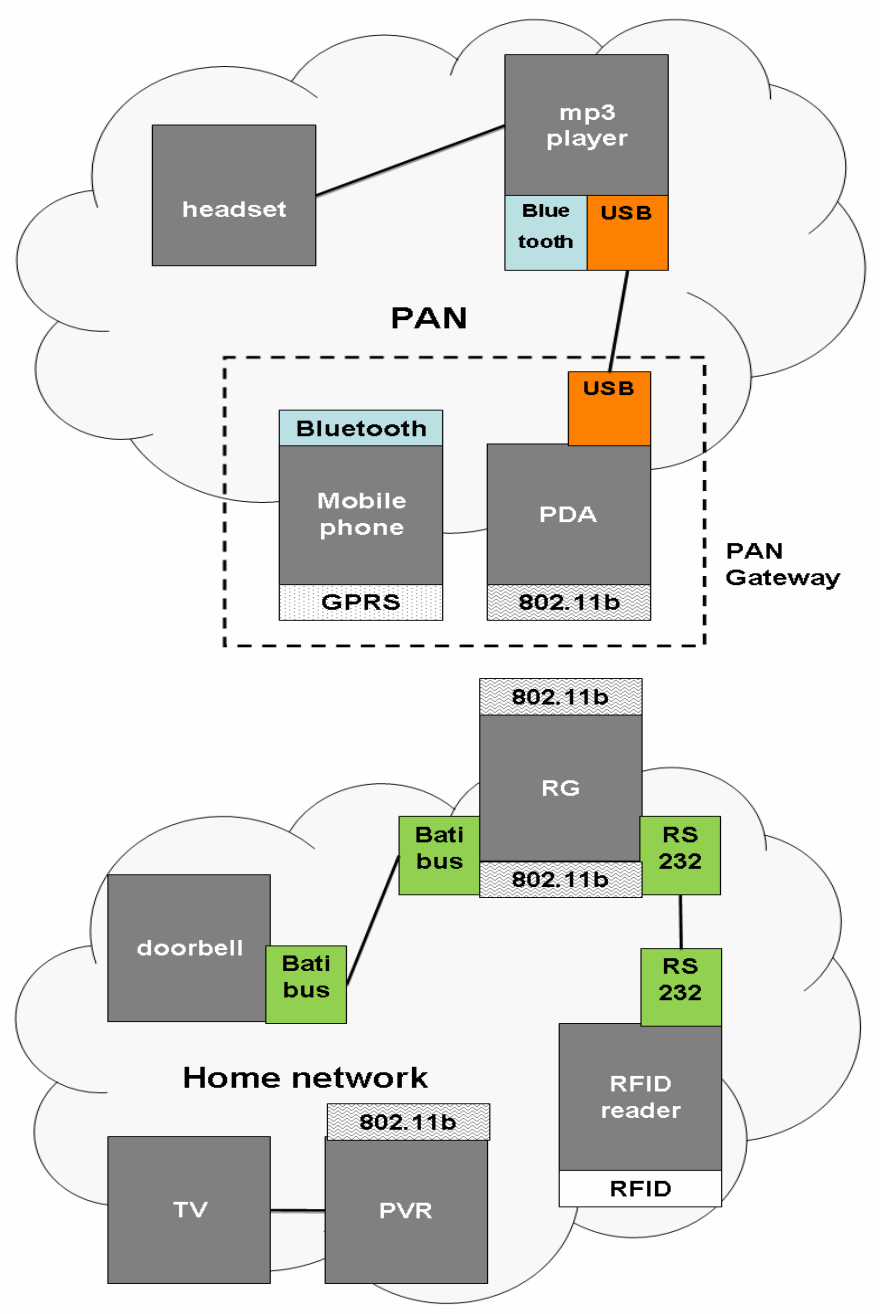

Figure 3. Architecture of the Always-at-Home demonstrator

A "who is at home" table is created by means of a MySQL [15] database on the RG, containing two tables: locations and users. The database is automatically updated by an RFIDreader application, implemented in Java, that interprets the RFID tags that the inhabitants of the home carry with them, and updates the database accordingly. When the doorbell rings, a Batibus-controller Java application on the RG queries the database. If nobody is at home, a video player (VideoLAN Client) is started on the PDA and the video is streamed from the front door camera to the PDA. If somebody is at home watching a PVR stream on, for instance, a TV, the PVR stream is paused, and the front door camera stream is forwarded to the PVR instead.

The most innovative part of the demonstrator is the contextaware configuration of the PN, in this particular case based on the interpretation of the RFID signals. The PNP role is completely fulfilled by the RG. Always at Home has not been trialed.

\section{MARCOPOLOG}

MarcoPolog focuses on PN-enabled location-based user services and user-friendly content management and is targeted at the tourism sector. The following use case is envisaged. Without any effort, a tourist collects a log of digital information about his trip, which he wants to show to his friends and family. He takes a lot of pictures, video- and sound- clips with his digital camera. He also collects digital information in museums, public places and tourist spots. He buys digital postcards and maintains a web log about his adventures, including an electronic agenda with the date and time of trips and events. The content is uploaded automatically to a server in his home network or to the server provided by his PNP. Which files are uploaded depends on synchronization rules, e.g. large pictures and video clips are only uploaded when the user has a cheap Wifi connection. The multimedia collection that is generated in this way is automatically managed and published by the PNP, who realizes the linking of pictures, video, documents, sound, agenda, text, location and other context information to create an appealing travel diary. The PNP takes care that, again based on synchronization rules, the content is replicated to other servers in the PN if necessary.

MarcoPolog's architecture is schematically drawn in Fig 4. The PAN is completely based on Bluetooth and comprises of a Universal Mobile Telecommunications System (UMTS) phone with integrated camera, a Global Positioning System (GPS) receiver, an IEEE $801.11 \mathrm{a} / \mathrm{b} / \mathrm{g}$-enabled PDA, and an RFID reader. The phone acts as the PAN gateway to the Internet. The scenario leads to a set of functional requirements for the service architecture:

- Content placed on the mobile phone (the PN Client) by any application is automatically detected and catalogued.

- A set of rules determines when which content is uploaded to a central server (the PN Repository).

- The PN Client keeps track of the content's status (metadata) so that content is only uploaded once. The meta-data is persistent, i.e. not lost when the device is switched off.

- The PN Repository can grant other users and applications access to the content.

In MarcoPolog's service architecture, all PN Clients of a user connect to a single PN Repository. Each PN Client runs a version of our PN synchronizer software which keeps track of all local content. The meta-data belonging to the content is transferred from each PN Client to the PN Repository on a regular basis. Consequently the PN Repository has knowledge of all files present on all PN Clients. The synchronizer software then decides which content is uploaded when to the repository based on a set of rules regarding content type and connectivity.

The architecture of the synchronizer software on the PN Client is shown in Fig. 5. It contains the following set of functional blocks: 


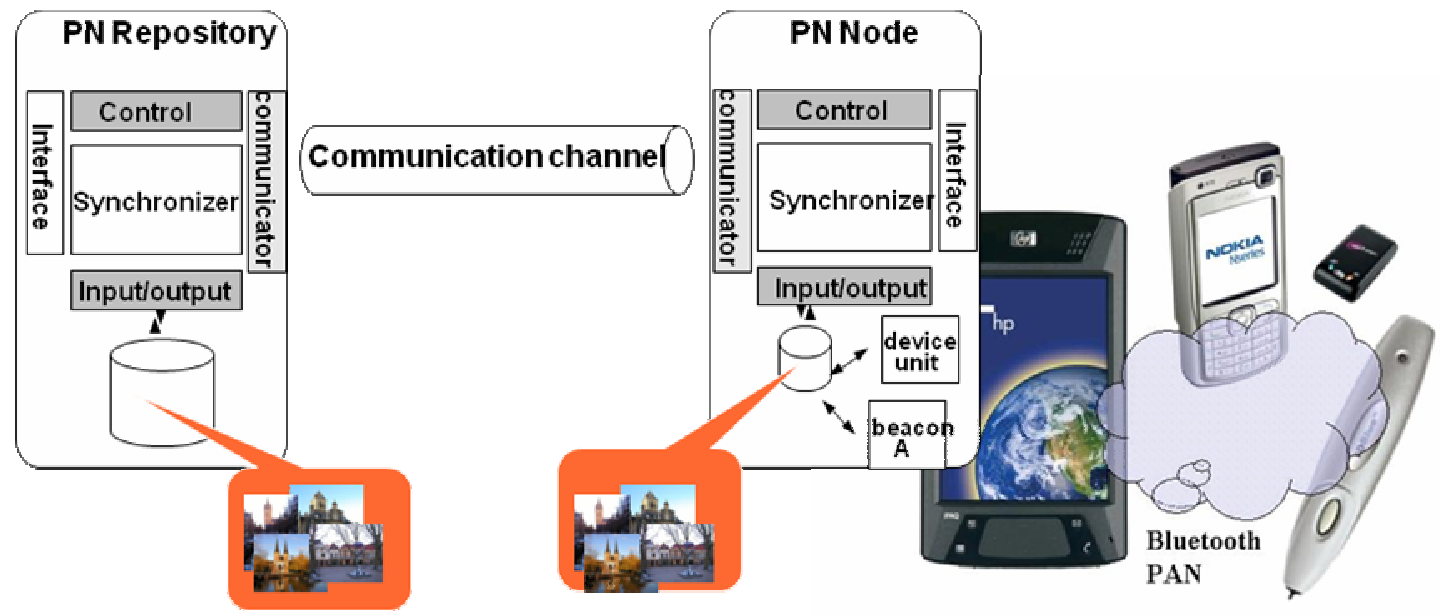

Figure 4. Architecture of the MarcoPolog demonstrator

- File Indexer: scans the file system of the PN Client, compares its finding with the file database, and updates the database as needed.

- File Database (DB): maintains all information about discovered files (name, size, type, etc.), and keeps track of the file's synchronization status.

- Sync Executor: scans the File DB and formulates a to-do list based on its findings. To-do tasks are either uploading file information (meta-data) or the actual content to the server.

- Communicator: scans the to-do list and decides which task to execute, based on its current connection with the PN Repository.

The PN Server runs the server version of the synchronizer software, containing the following set of functional blocks:

- SQL interface: provides access to the SQL database on the server, which persistently stores the meta-data from all PN Clients, including a link to the file's location on the server's file system after the file has been uploaded.

- Access Control: decides if an incoming request to content in the server database is granted.

Communication between PN Clients and the PN Repository is based on Hyper Text Transfer Protocol (HTTP) Post messages.

The main innovation of MarcoPolog is the automatic synchronization of the generated content. The tourist can fully concentrate on collecting pictures, videos, web pages, coordinates, etc, using any application that is available on his device. All content is stored on the mobile phone. The complete route is recorded using continuous location tracking, and the generated content is tagged to the route using timestamp comparison. After synchronization (i.e. the upload), the content is also available on the server. Synchronization only happens when the PAN is connected to a network. In the mean while, the file system of the phone is used as a cache. A travel log displaying the content is automatically generated by the server, using an interactive geographical map (Google Maps). Also innovative is the use of RFID technology to label content in case no GPS coverage is available or when additional information of an object is supplied by way of a reference to a web page (see Fig. 6).

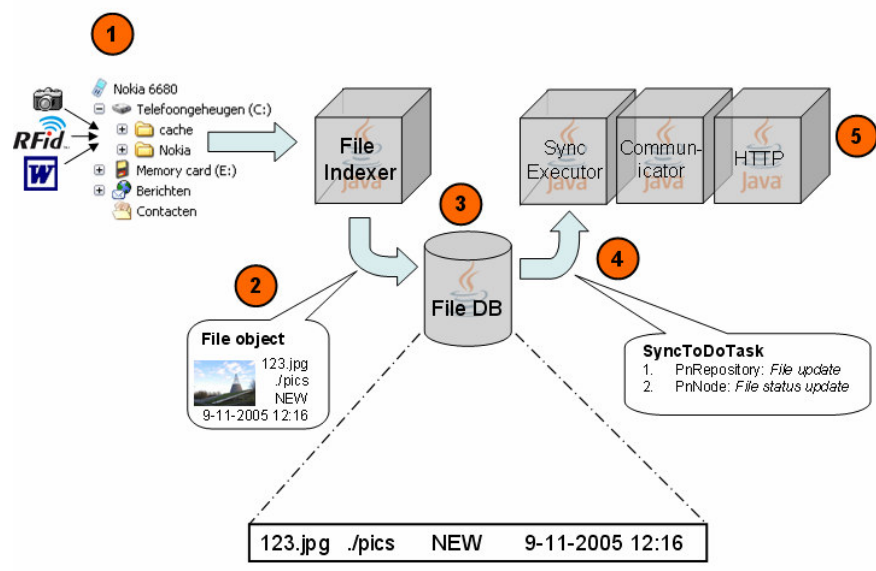

Figure 5. Client-based PN Synchronizer architecture

We have performed user trials with two target groups (young professionals and teenagers), and collaborated with three museums in The Hague, The Netherlands. Next to the research methods used with the Medicam pilot, we also used experience sampling and probes. For experience sampling we used a phone-based tool developed by the Freeband TUMCAT project [16]. The most interesting results of the user trials are summarized below:

- Battery stress. All users found the daily recharging of the batteries of all PAN devices stressful.

- Connection stress. It was not always sure if all Bluetooth connections were up and running, if there was a GPS lock and if UMTS coverage was available. 


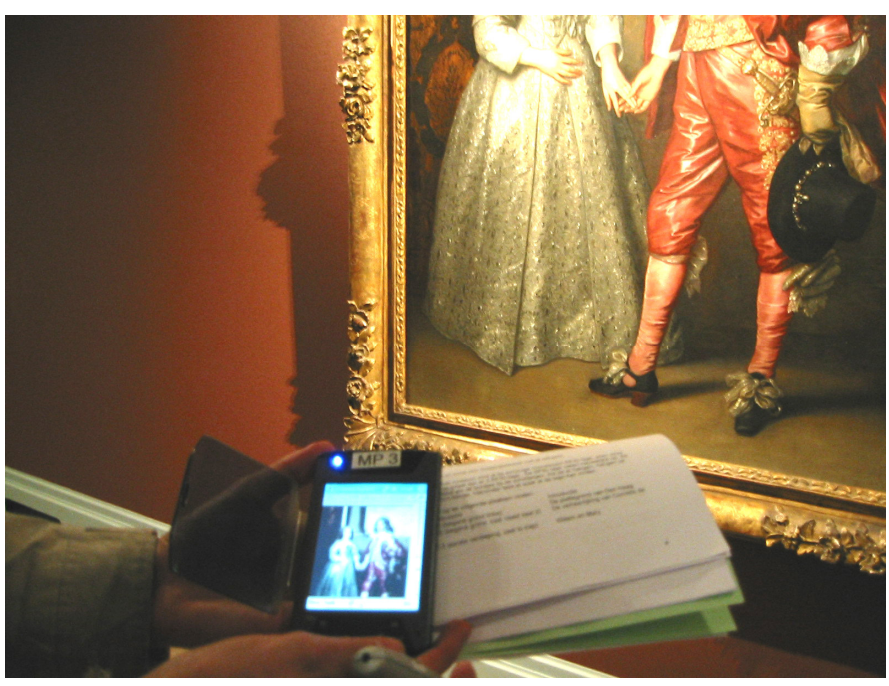

Figure. 6 A pilot user reading the information about a painting obtained via an RFID tag.

- It is not always easy and comfortable to carry around so many PAN devices (and their power adapters).

- All users saw a clear advantage in PNs offering easy access to and storage of content. Some people did not feel comfortable with the central storage of content at the premises of a commercial PNP from a security and privacy point of view.
- Teenagers were afraid of losing or breaking all those expensive gadgets.

- Young professionals were especially intrigued by the automatic upload and the RFID tagging and liked the (b)logging functionality for holidays and sharing with friends.

\section{PNPAY TRAVEL}

Our most recent demonstrator deals with applying PNs to achieve easy and differentiated billing services. In this paper we limit ourselves to the use case and the general architecture, because the user trial is still ongoing.

MarcoPolog taught us that a PN can collect a lot of data about the context of the user. The idea of PNPay is that the PN can send relevant data to service providers so that they can generate a correct bill for services that require payment. Crucial is that the PN sends each service provider only the minimum set of data necessary for billing, and as such maximizes the privacy of the user. If the billing provider and service provider are different parties, this data does not need to contain the identity of the user, assuming that the billing party is trusted and the only one containing ID information. The shear mountain of information present in the PN enables a so far unimaginable high level of differentiating accounting services.

PNPay Travel shows an example of such easy billing

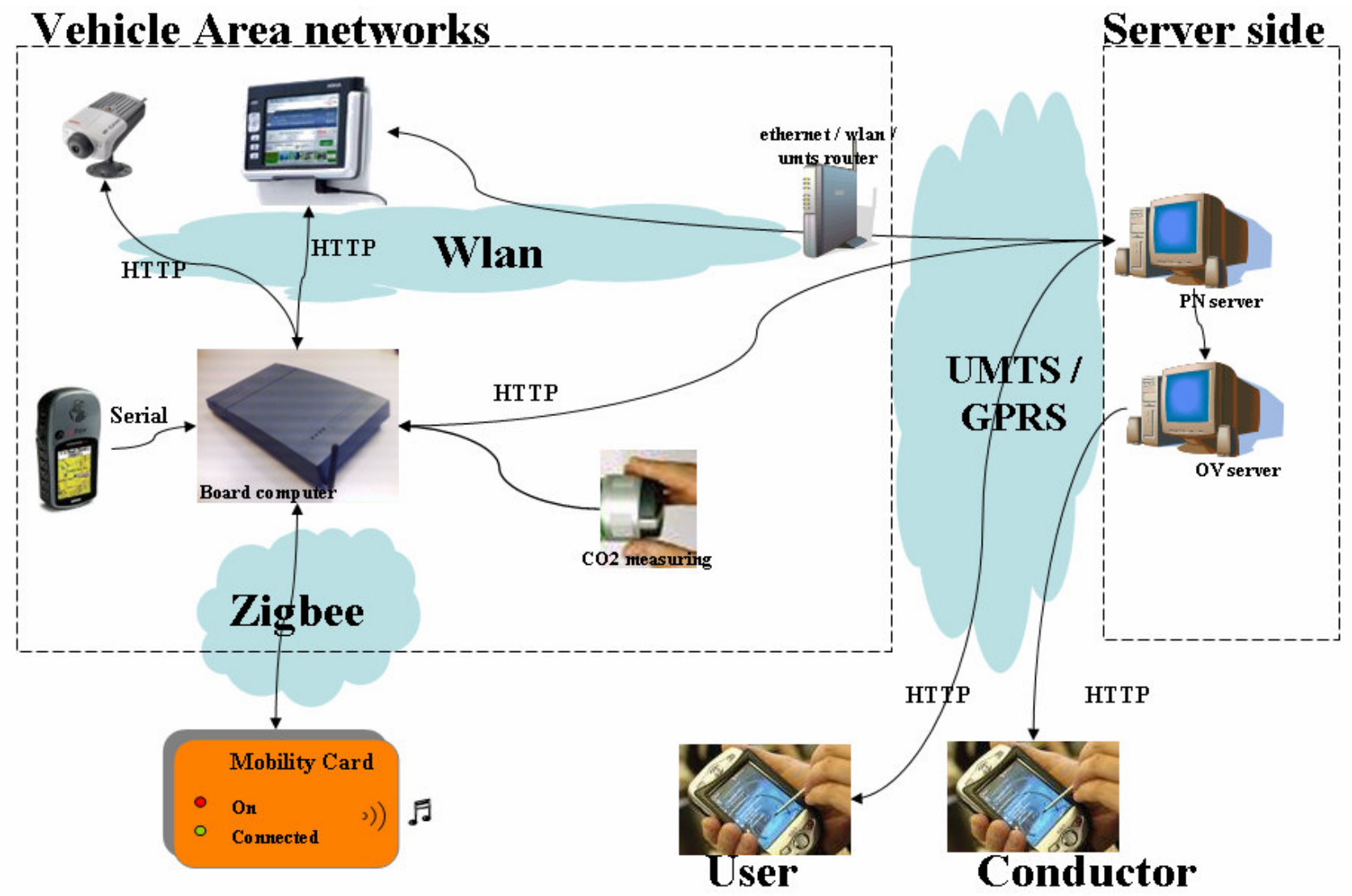

Figure 7. PNPay Travel network architecture 
services. It combines road pricing with public transport ticketing in an intuitive fashion. Furthermore, it combines road navigation suggestions with public transport time tables. So, PNPay Travel transforms single-sector based travel arrangements into multi-sector mobility arrangements: "getting there on time using any transport". Here is the use case that we are currently trialing: When traveling, the user always carries a personal credit-card sized Mobility Card around. He wants to go from A to B. He gets into his car. The car gateway authenticates him. He enters his destination into the navigation system, which calculates the quickest or the cheapest route, depending on the his preference. The navigations system includes public transport in the calculations. During the drive the navigation system keeps the traveler informed about the costs he is making, based on the road pricing rates and a tax on the pollution that the car generates: $50 \mathrm{mph}$ in fifth gear is cheaper than in fourth gear!. The navigation system detects an unexpected traffic jam ahead and decides that it is best to park the car at the nearest train station and continue the trip by public transport. While in the train, the billing is again based on the traveler's location data, but now accounted for public transport rates. Buying a ticket is not needed: the mobility card is detected automatically. The user is now provided feedback to his PDA.

The PNPay Travel network architecture is shown in Fig. 7. The center of the vehicle area network (either car- or train network) is the on-board computer or vehicle area network gateway. It is based on the Unattended Autonomous Surveillance (UAS) system developed for tele-care and telemonitoring applications [17]. It detects the presence of a Mobility Card by means of IEEE 802.15.4 (Zigbee) technology. Further authentication of the driver is done with a face validation system as described in [18]. A tablet PC together with a GPS receiver forms a very basic navigation system. In the train only the gateway is present. The gateway collects the data from the camera, the navigation system, and the mobility card, and sends them to the traveler's PN Server via UMTS or GPRS. The gateway also receives data from the pollution meter, which can be used for adapting the road pricing tariffs. The PN Server analyzes all data and provides feedback to the tablet PC (when in the car) or, with a simplified user interface, to the mobile phone of the traveler (when in the train). The PN Server sends the minimum amount of data needed for billing to the service provider, which can be checked by a ticket inspector and the traveler himself. Fig. 8 shows the front seat and the boot of the car used in the user trial.

\section{CONCLUSIONS AND FUTURE WORK}

We designed four demonstrators: Medicam, Always at Home, MarcoPolog and PNPay Travel for the healthcare, travel, tourist and transport sectors respectively. By developing these demonstrators and performing small scale user trials we encountered both opportunities and pitfalls in deploying PNs on a commercial basis. The functional components of the demonstrators include service and device discovery and control, content management, context-aware services, security, and billing services. On each of these functions, the main conclusions can be summarized as follows.
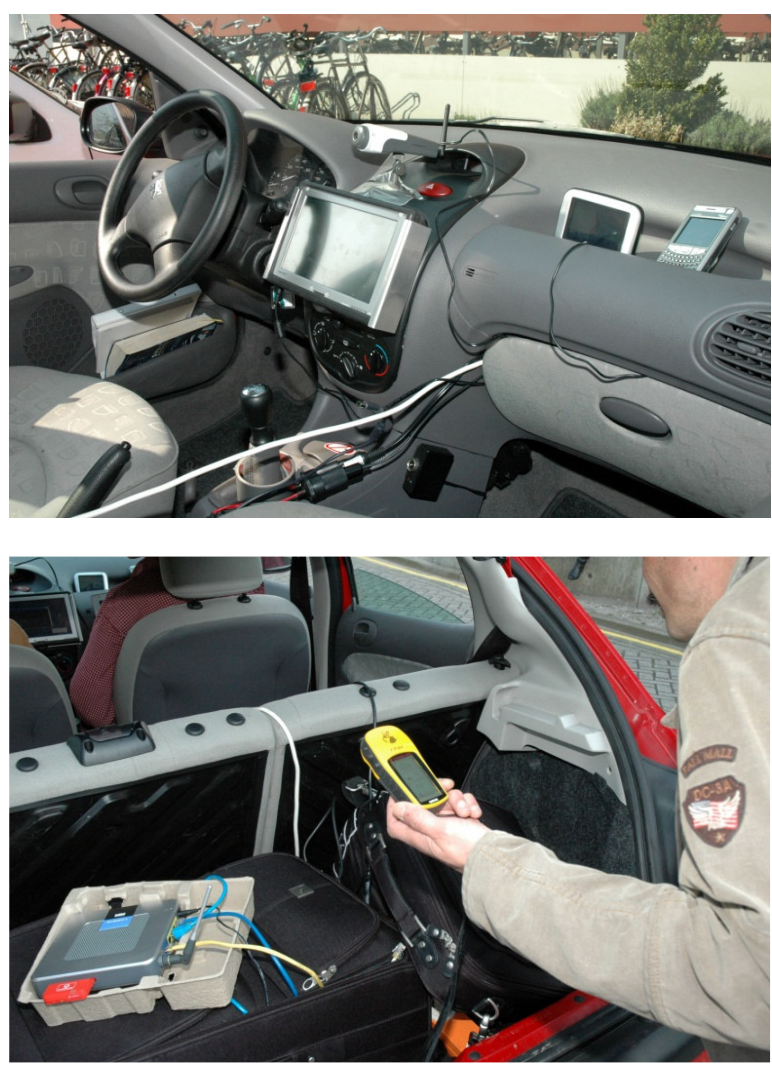

Figure 8. Top: The dashboard of the car. In the middle, the tablet PC with the authentication camera on top. To the right, a commercially available navigation system and the user's PDA. Bottom: The car area network gateway and the GPS receiver.

Current service and device discovery and control protocols are not at all suitable for PNs. They are constructed for use in a single small-scale private network only. Extending their reach to other private networks, either by tunneling or by constructing an overlay network on the application layer, results in discovery times that are often too long [19]. Furthermore, operation in PNs require some properties to be discovered that are not supported by current discovery protocols, such as the costs involved or the quality of the service to be expected when accessing the service at a particular moment. Finally, discovery should be extended to services offered to the PN by third-party service providers, and for the user it should always be clear if a specific service is available within the $\mathrm{PN}$ or is offered to the $\mathrm{PN}$. We are currently investigating if the IP Multimedia Subsystem (IMS [20]) can be used to provide this extra discovery functionality to the PN.

There is a clear need for an intelligent content management system within the PN. The more devices a PN consists of, the less a user should be required to invent his own rules and remember where certain content is stored and how it can be accessed. Moreover, these rules are often dependent on the user's context. This problem cannot be solved by centralizing the storage of all content on a single server in the PN. The user may not trust such an architecture from a security and privacy point of view. We have designed a distributed architecture that overcomes these issues. At the moment, we are studying how 
this architecture can be improved using existing peer-to-peer technologies such as Tribler [21]. Tribler introduces the notion of "friends" to peer-to-peer networks. This can be applied to PNs when the various content servers and clients within a PN are labeled as "friends". We are also designing an application, the "PN Explorer", that provides an intuitive user interface for the content present in the PN, regardless of where it is stored.

A distributed PN content management architecture requires free replication of content within the PN and secure access to content from outside the PN. This revives the concept of Authorized Domains, as proposed by the Digital Video Broadcast (DVB) Forum, elaborated for home networks by Popescu et al [22], and partly standardized in the Open Mobile Alliance version 2 (OMA V2) Digital Rights Management (DRM) working group [23]. We have not addressed this topic yet. However, the first step in PN security, before content sharing becomes an issue, is the authentication of physical access to the PN devices by the PN owner. We have used face validation to authorize the incorporation of an in-car network into the user's PN.

Our four demonstrators each show a spectrum of examples of context awareness. Most of them are location-based services, which can be categorized as either location-based user services or location-based $P N$ configuration services. Examples of user services are the application of GPS coordinates for generating travel logs, navigation suggestions and billing data, as well as the use of RFID tags to acquire local tourist information. Examples of PN configuration services comprise are the use of RFID signals to find out who is at home and consequently route camera images to the correct video player, and the detection of a Zigbee train- or car network to present billing information to the correct device with the right user interface. The latter category of contextaware services is specific to PNs and makes the PN as a whole context aware [24].

Finally, we have shown that PN service platforms can be used to improve existing services and enable new services in a large variety of business and social sectors. A PN service platform enables providers to offer services to seemingly very different customers without having to invest in different technology. This is new, because many sectors, and in particular public sectors such as health care and public transport, have a history of developing their own dedicated stove-pipe solutions for similar applications. Furthermore, PN service platforms provide an opportunity to develop truly transsector services [25]. In PNPay Travel we overcame the barrier between the public transport sector and the private transport sector by integrating billing services and combining road navigation with public transport schedules. The trials that are currently being performed should indicate how appealing this will be to the user.

The user experiments we have performed so far show that PNs can offer great ease of use of ICT services to the owner of the PN. However, the heterogeneity and dynamics of PNs put stringent requirements on the dependability of the connectivity and the speed of (re-)connecting to the various networks. Our user trials show that these requirements are not met by the current technology. Slow configuration and errors caused the users to be confronted with technology details, whereas PNs were meant to hide those.

\section{REFERENCES}

[1] H.B.J. ter Harmsel, and N.H.G. Baken, "Broadband services on rail tracks; the business case for fast-roaming WLAN", Proceedings of the $45^{\text {th }}$ FITCE Congress, Athens, 2006.

[2] F.T.H. den Hartog, M. Balm, C.M. de Jong, and J.J.B. Kwaaitaal, "Convergence of Residential Gateway technology", IEEE Commun. Mag , vol. 42, pp. 138-143, May 2004.

[3] I.G. Niemegeers and S.M. Heemstra de Groot, "From Personal Area Networks to Personal Networks: A user oriented approach", Wireless Personal Communications, vol. 22, pp. 175-186, August 2002.

[4] http://pnp2008.freeband.nl.

[5] http://www.bluetooth.com.

[6] ANSI/EIA 709.1-4, EN 14908, http://www.lonworks.org.

[7] EN 50090, http://www.knx.org.

[8] E. R. Fledderus, O. D. Rietkerk, F. T. H. den Hartog, I. G. M. M. Niemegeers, and S.M. Heemstra de Groot, "Toward viable Personal Networks and Fednets - a value-web perspective", Wireless Personal Communications, vol 38, pp. 103-115, June 2006.

[9] F.T.H. den Hartog, J.R. Schmidt, and A de Vries, "On the potential of Personal Networks for hospitals", International Journal of Medical Informatics, vol. 75, pp. 658-663, 2006.

[10] http://www.ietf.org/rfc/rfc3927.txt .

[11] Push-to-Talk on a mobile phone, GSA opinion paper, http://www.gsacom.com/downloads/pdf/gsa_opinion_paper_015.php4 .

[12] http://www.ietf.org/rfc/rfc3288.txt .

[13] F.T.H. den Hartog and M.E. Peeters, "A concrete example of a Personal Network architecture", Proceedings of the $3^{\text {rd }}$ Consumer Communications and Networking Conference (CCNC2006), pp. 514518, January 2006.

[14] http://www.rfid.org.

[15] http://www.mysql.com

[16] A. Vermeeren and J. Kort, "Developing a testbed for automated user experience measurement of context aware mobile applications", Proceedings of the The Second COST294-MAUSE International Open Workshop: User Experience - Towards a Unified View, Oslo, 2006.

[17] Ari Andersin et al, "D1.2.22 Public Final Report of the IST 2001-37422 project INMOVE", March 2005.

[18] Qian Tao and Raymond N. J. Veldhuis, "Biometric authentication for a mobile personal device", Proceedings of the $1^{\text {st }}$ International Workshop on Personalized Networks (Pernets2006), San Jose, July 2006.

[19] Majid Ghader, Rasmus L. Olsen, Venkatesha Prasad, Martin Jacobsson, Luis Sanchez, Jorge Lanza, Wassef Louati, Marc Girod Genet, Djamal Zeghlache, Rahim Tafazolli, "Service Discovery in Personal Networks; Design, Implementation and Analysis", Proceedings of the 15th IST Mobile \& Wireless Communications Summit, Myconos, June 2006.

[20] http://www.3gpp.org.

[21] J. Pouwelse, P. Garbacki, J. Wang, A. Bakker, J.Yang, A.Iosup, D.Epema, M.Reinders, M. van Steen, and H.Sips, "Tribler: A SocialBased Peer-to-Peer System", 5th International Workshop on Peer-toPeer Systems (IPTPS'06), Feb 2006.

[22] Bogdan C. Popescu, Bruno Crispo, Frank L.A.J. Kamperman, and Andrew S. Tanenbaum, Proceedings of the 4th ACM workshop on Digital rights management, pp. 1 - 10, 2004 .

[23] http://www.openmobilealliance.org/tech/wg_committees/DRM.html.

[24] Rachid Ait Yaiz, Franklin Selgert and Frank den Hartog, "On the definition and the relevance of context-awareness in Personal Networks", Proceedings of the $1^{\text {st }}$ International Workshop on Personalized Networks, San José, July 2006.

[25] Nico Baken, Nico van Belleghem, Edgar van Boven, and Annemieke de Korte, "Unravelling 21 st century riddles -universal network visions from a human perspective", The Journal of The Communications Network, Vol.5, pp. 11-20, October-December 2006. 